\title{
Frequency Analysis of the RRc Variables of the MACHO Database for the LMC
}

\author{
G. Kovács ${ }^{13}$, C. Alcock ${ }^{1}$, R. Allsman ${ }^{2}$, D. Alves ${ }^{3}$, T. Axelrod ${ }^{2}$, \\ A. Becker ${ }^{4}$, D. Bennett ${ }^{12}$, C. Clement ${ }^{15}$, K. H. Cook ${ }^{1}$, A. Drake ${ }^{2}$, \\ K. Freeman ${ }^{2}$, M. Geha ${ }^{1}$, K. Griest ${ }^{5}$, D. W. Kurtz ${ }^{14}$, M. Lehner ${ }^{11}$, \\ S. Marshall ${ }^{1}$, D. Minniti ${ }^{6}$, C. Nelson ${ }^{7}$, B. Peterson ${ }^{2}$, P. Popowski ${ }^{1}$, \\ M. Pratt ${ }^{4}$, P. Quinn ${ }^{8}$, A. Rodgers ${ }^{16}$, J. Rowe ${ }^{15}$, C. Stubbs ${ }^{4}$, \\ W. Sutherland ${ }^{9}$, A. Tomaney ${ }^{4}$, T. Vandehei ${ }^{5}$, D. L. Welch ${ }^{10}$ \\ (The MACHO Collaboration)
}

\begin{abstract}
We present the first massive frequency analysis of the 1200 first overtone RR Lyrae stars in the Large Magellanic Cloud observed in the first $4.3 \mathrm{yr}$ of the MACHO project. Besides the many new doublemode variables, we also discovered stars with closely spaced frequencies. These variables are most probably nonradial pulsators.
\end{abstract}

\section{Variables with Close Frequency Components}

The MACHO variable star database enables us to investigate many problems related to pulsating stars. One of these is the question of the occurrence of amplitude-modulated (i.e. Blazhko-type) variables among first overtone $R R$ Lyrae (RRc) stars. Until now, this question has not been answered mostly because of the lack of proper observational data. Based on the frequency analysis of the MACHO RRc sample of 1200 stars in the LMC, the present work is the first systematic study of this problem. The detailed description of our results extended on an even longer dataset will be published elsewhere (Alcock et al., 2000).

On the basis of standard Fourier frequency analysis, we found $74 \%$ of the variables to be monoperiodic. Defining Blazhko variables as those exhibiting

\footnotetext{
${ }^{1}$ IGPP, Lawrence Livermore National Laboratory, Livermore, CA 94550, USA

${ }^{2}$ Mount Stromlo and Siding Spring Observatory, ANU, Weston Creek, ACT 0200, Australia

${ }^{3}$ Space Telescope Science Institute, Baltimore, MD 21218, USA

${ }^{4}$ Department of Astronomy, University of Washington, Seattle, WA 98195, USA

${ }^{5}$ Department of Physics, University of California, San Diego, CA 92093, USA

${ }^{6}$ Department Astronomia, Universidad Catolica, Chile

${ }^{7}$ Department of Physics, University of California, Berkeley, CA 94720, USA

${ }^{8}$ European Southern Observatory, Garching, D-85748, Germany

${ }^{9}$ Department of Physics, Oxford University, OX1 3RH, England

${ }^{10}$ Department of Physics and Astronomy, McMaster University, Hamilton, L82 4M1, Canada

${ }^{11}$ Department of Physics, University of Sheffield, S3 7RH, England

${ }^{12}$ Department of Physics, University of Notre Dame, South Bend, IN 46556, USA

${ }^{13}$ Konkoly Observatory, P.O. Box 67, H-1525 Budapest, Hungary

${ }^{14}$ Department of Astronomy, University of Cape Town, Rondebosch 7701, South Africa

${ }^{15}$ Department of Astronomy, University of Toronto, Toronto, M5S 3H8, Canada

${ }^{16}$ deceased
} 


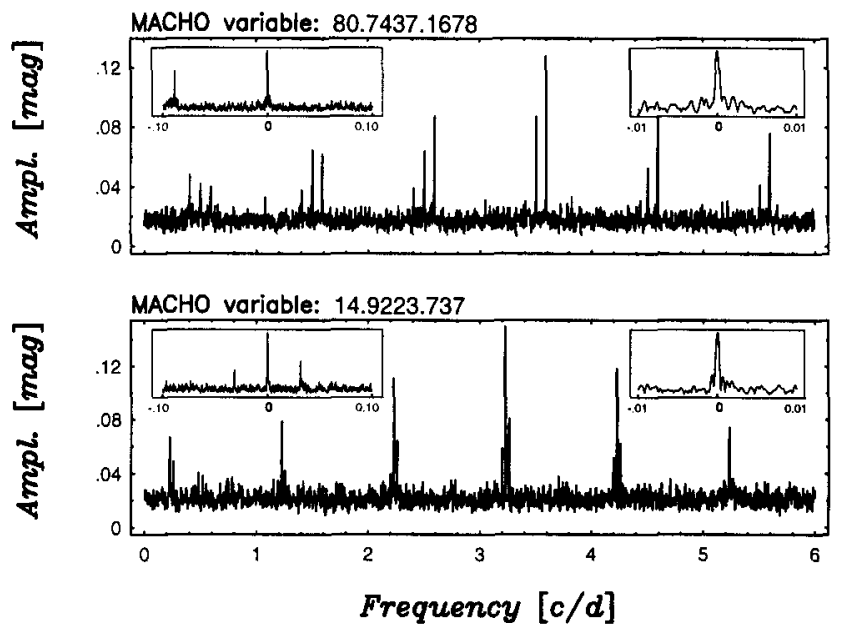

Figure 1. Two examples of the RRc stars in LMC with closely spaced frequencies. The insets show the fine structure around the main peaks.

equidistant triplets in their frequency spectra, we discovered 17 such stars. In addition, we also found 27 stars with 2 closely spaced frequencies. Other studies, very recently, have also discovered some representatives of this type of variable (in M55 by Olech et al. (2000) in the OGLE sample of the Galactic bulge by Moskalik (2000)). (The apparent triplet structure in the low-frequency regime in the upper panel is due to power leakage from negative frequencies.) In Fig. 1 we show representatives of the above classes of variables. The most natural explanation of the occurrence of variables with closely spaced frequencies is the presence of nonradial modes in those stars. Recent theoretical models lend further support to this suggestion (Dziembowski \& Cassisi 2000). Furthermore, the number of RRc Blazhko stars is rather low. If we compare their frequency with the $\approx 20 \%$ occurrence rate of their relatives among the fundamental mode RR Lyrae stars, we can conclude that:

Magnetic oblique rotator models which assume the presence of magnetic field as the underlying cause of amplitude modulation are not easy to maintain, because of the difficulties in understanding how the role of magnetic field becomes so much less important as we move from the fundamental to the first overtone variables.

Acknowledgments. The supports of the following grants are acknowledged: OTKA T024022, T026031, and T030954.

\section{References}

Alcock, C. et al. 2000, in preparation

Dziembowski, W. A. \& Cassisi, S. 2000, in these proceedings, p. 321

Moskalik, P. 2000, in these proceedings, p. 313

Olech, A., Kaluzny, J., Thompson, I. B., et al. 2000, in these proceedings, p. 317 